\title{
Repeated triggering of sporulation in Bacillus subtilis selects against a protein that affects the timing of cell division
}

\author{
Jeroen Siebring ${ }^{1}$, Matthijs $\mathrm{JH}_{\mathrm{Elema}}{ }^{1}$, Fátima Drubi Vega ${ }^{2,3}$, Ákos T Kovács ${ }^{1,5}$, \\ Patsy Haccou ${ }^{2,3}$ and Oscar P Kuipers ${ }^{1,4}$ \\ ${ }^{1}$ Molecular Genetics Group, Groningen Biomolecular Sciences and Biotechnology Institute, University of \\ Groningen, Groningen, The Netherlands; ${ }^{2}$ Institute of Environmental Sciences (CML), Leiden University, \\ Leiden, The Netherlands; ${ }^{3}$ Leiden University College the Hague, Leiden University, The Hague, \\ The Netherlands and ${ }^{4}$ Kluyver Centre for Genomics of Industrial Fermentation, Delft, The Netherlands
}

\begin{abstract}
Bacillus subtilis sporulation is a last-resort phenotypical adaptation in response to starvation. The regulatory network underlying this developmental pathway has been studied extensively. However, how sporulation initiation is concerted in relation to the environmental nutrient availability is poorly understood. In a fed-batch fermentation set-up, in which sporulation of ultraviolet (UV)-mutagenized $B$. subtilis is repeatedly triggered by periods of starvation, fitter strains with mutated tagE evolved. These mutants display altered timing of phenotypical differentiation. The substrate for the wall teichoic acid (WTA)-modifying enzyme TagE, UDP-glucose, has recently been shown to be an intracellular proxy for nutrient availability, and influences the timing of cell division. Here we suggest that UDP-glucose also influences timing of cellular differentiation.
\end{abstract}

The ISME Journal (2014) 8, 77-87; doi:10.1038/ismej.2013.128; published online 8 August 2013

Subject Category: Evolutionary genetics

Keywords: evolution; Bacillus subtilis; tagE; UDP-glucose; sporulation

\section{Introduction}

Next-generation sequencing has revolutionized experimental evolution as a tool for studying the molecular basis of evolutionary adaptations on a genome scale (Barrick et al., 2009; Blount et al., 2012). Experimental evolution has successfully been used for studying, amongst others, cellular decision making. For example, by using alternating selection pressures, bacterial strains with altered phenotypic heterogeneity were selected (Beaumont et al., 2009; Rainey et al., 2011). Both timing of differentiation and the fraction of the population that pursues a specific developmental pathway determines the fitness of a bacterial strain for a particular environment.

$B$. subtilis endospore formation is a last-resort adaptive response to starvation (Lemon et al., 2000; Sonenshein, 2000; Veening et al., 2005) and an ideal

Correspondence: OP Kuipers, Molecular Genetics Group, Groningen Biomolecular Sciences and Biotechnology Institute, University of Groningen, Nijenborgh 7, Groningen 9747 AG, The Netherlands.

E-mail: o.p.kuipers@rug.nl

${ }^{5}$ Present address: Terrestrial Biofilms Group, Institute of Microbiology, Friedrich Schiller University Jena, Jena, Germany. Received 1 February 2013; revised 10 June 2013; accepted 3 July 2013; published online 8 August 2013 system for studying cellular decision making. The sporulation decision is bimodal and irreversible, and spores can be discriminated from vegetative cells both visually and based on stress resistance. Our experimental evolution study aimed to find yet unidentified elements involved in timing and distribution of sporulation initiation in $B$. subtilis (Maughan and Nicholson, 2004; Veening et al., 2008).

Rapid phenotype switching between vegetative cells and spores was pursued by introducing starvation episodes to cultures grown in chemically defined sporulation medium (CDSM) in a fed-batch set-up. Starvation episodes were accomplished by sequentially feeding discrete amounts of glucose at intervals long enough for the offered glucose to be fully consumed and starvation to occur. Feeding intervals of $4 \mathrm{~h}$ could initiate sporulation, but were short enough for vegetative cells to potentially survive, in an attempt to apply selection pressure on the decision to initiate sporulation. Additionally, it was anticipated that then randomizing glucoseaddition intervals would result in different selection pressures. This could provide information on how $B$. subtilis encodes the coordination of sporulation while the future of its environment is uncertain. Strains, randomly mutagenized by ultraviolet (UV)-radiation treatment, were cultivated in these 
fluctuating environments. Fitness of evolved strains was determined by competition experiments (Gordo et al., 2011) and the genomic adaptations of fitter strains were studied by whole-genome sequencing.

We expected to find mutants with altered spore accumulation over time: initiated by environmental cue and/or steeper with regular glucose additions (deterministic) versus stochastic and/or more gradual upon irregular glucose additions (bet-hedging). Selection of strains with increased sporulation efficiency is not anticipated. A previous study showed that sporulation efficiency is robust even with strong selection pressure for sporulation (Maughan and Nicholson, 2004). Mutations on several regulatory levels were expected, for instance, mutations resulting in altered sensing of nutrient availability (for example, by mutations causing altered substrate affinity of an importer complex), altered sigma factor binding that resulted in changed network sensitivity or mutations altering metabolic fluxes (for example, loss of enzyme function altering nutrient distributions).

Here, we show that losing the seemingly redundant enzyme TagE, which glycosylates glycerol phosphate subunits of major wall teichoic acid (WTA), increases the fitness of $B$. subtilis in the selection environment and alters the timing of cell differentiation.

\section{Materials and methods}

Detailed Materials and methods can be found in the Supplementary Information (SI1).

\section{Media}

CDSM was chosen for our evolution experiments because it is chemically defined and supports high sporulation efficiency of $B$. subtilis. CDSM was prepared as described previously (Hageman et al., 1984; Veening et al., 2006). Up to $20 \mathrm{~mm}$ of D-glucose was added as the sole carbon source when appropriate.

\section{Analysis of spore accumulation in CDSM-grown}

B. subtilis cultures

B. subtilis 168 was grown in a shake flask in CDSM initially supplemented with $20 \mathrm{~mm}$ glucose. Fresh CDSM was inoculated with $0.1 \%$ (v/v) of CDSMgrown overnight culture. The culture was shaken at $37^{\circ} \mathrm{C}$. The distribution of live cells, dead cells and phase-bright spores throughout the cultivation was determined using ethidium bromide and (fluorescence) microscopy. Cells with ethidium bromidestained chromatin were recorded as dead, as they cannot, like live cells, actively export ethidium bromide. The glucose concentration in samples was determined using a D-Glucose assay (Boehringer Mannheim, Mannheim, Germany).
Batch fermentations with sequential glucose-feeding regimes

Batch fermentations were performed using Multifors bioreactors (Infors HT, Bottmingen, Switzerland). $\mathrm{pH}$ and $\mathrm{pO}_{2}$ (dissolved oxygen) were monitored in real time but not controlled throughout the cultivation in order to mimic shake-flask cultivations.

CDSM for batch fermentations lacked glucose and was supplemented with $0.03 \%$ antifoam (Struktol J673) and $20 \mathrm{~mm}$ of L-alanine. Germinant L-alanine was added to the medium for stimulating spore germination in order to prevent that altered germination becomes the major beneficial property in evolved mutants (Supplementary Information SI6).

Twenty discrete portions of CDSM containing $40 \%$ glucose were fed at intervals of $4 \mathrm{~h}$, each resulting in addition of $1 \mathrm{~mm}$ glucose (final concentration). The feed pump mounted on the bioreactor system was used for the additions of high-glucose CDSM and was externally controlled by the manufacturer's software (Iris, Infors; see Supplementary Information SI3).

For erratic glucose feeding, 20 additions were programmed in the same total time frame of $80 \mathrm{~h}$ but with randomized feeding intervals.

Competition experiments were inoculated with mid-exponentially growing CDSM cultures (optical density (OD) ${ }_{600} 0.6-0.8$ ) or dense spore crops, mixed 1:1 based on $\mathrm{OD}_{600}$. Endpoint samples were taken at $144 \mathrm{~h}$ ( $64 \mathrm{~h}$ after the final glucose addition) when only spores remain, except for competitions between stJS01 and the non-sporulating $\Delta \operatorname{sig} F$ strain, when the last sample was taken before the final glucose addition.

Each sample $(1 \mathrm{ml})$ taken from a bioreactor was used for (1) determining $\mathrm{OD}_{600}$, (2) (fluorescence) microscopy, (3) species colony count (in case of competition experiments) and (4) spore counts (plate assay)(Supplementary Figure S1).

\section{Selection and isolation of mutants with favorable mutations}

stJS03 (Supplementary Table S3) was used for evolution experiments because it allows the study of sporulation initiation via the incorporated fluorescent proteins $\left(\mathrm{P}_{s s p E-2 \mathrm{G}}-g f p, \mathrm{P}_{\text {spoIIA-mCherry). spoIIA }}\right.$ is one of the first genes to be expressed (in the mother cell) after initiation of sporulation (Errington, 1993; Hoch, 1993; Fujita et al., 2005; Veening et al., 2005), and thus $\mathrm{P}_{\text {spoIIA-mCherry can }}$ serve as a reporter for commitment to sporulation. $s s p E$ is expressed in spores and its promoter can therefore be used for fluorescent labeling of spores, allowing discrimination between $\mathrm{GFP}^{+}$and $\mathrm{GFP}^{-}$ spores in competitions (Webb et al., 1995). Both constructs are stably integrated in the chromosome.

Ancestral strain stJS03 was UV-mutagenized before the selection experiments (Figure 1, stage 1; 


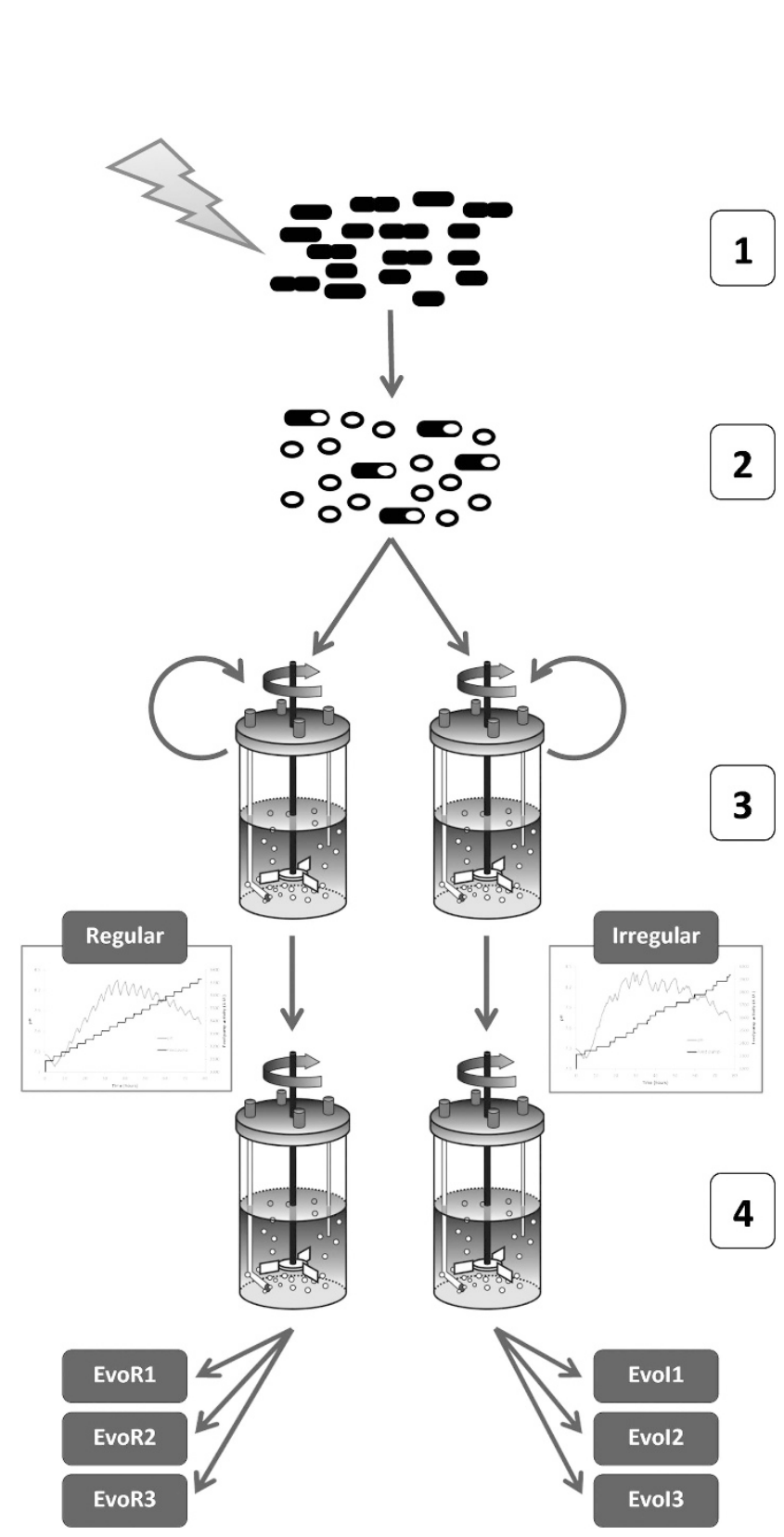

Figure 1 A graphical representation of the evolution process and strain fitness assessment leading to the isolation of evolved strains. (Stage 1) Random mutagenesis: Vegetative stJS03 cells are being radiated with a range of different UV dosages. (Stage 2) Sporulation: The UV-radiated culture is left to form spores in order to lose potential sporulation-negative phenotypes. (Stage 3) Selection: Enriching fitter strains was initiated by inoculating the bioreactors with UV-mutagenized stJS03 spores only. After 20 glucose additions, glucose influx was stopped and the culture was left at $37^{\circ} \mathrm{C}$ while being properly aerated. From the resulting dense spore crop an aliquot was extracted of which $15 \mathrm{ml}$ was used for inoculation for the second round of selection. From the resulting spore crop of the second selection cycle $100 \mathrm{ml}$ was extracted and stored for future use. (Stage 4) Competition and mutant isolation: A sample of the preceding consecutive selection rounds is co-inoculated with stJS02 in the respective environments to identify emergence of fitter mutants. From these competitions, at three stages, samples were taken and $\mathrm{Cm}^{\mathrm{R}}$ cells were isolated-EvoR1-3 from the environment with the regular glucose influxes and EvoI1-3 from the environment with irregular glucose additions-for whole-genome sequencing.

Supplementary Table S3; Supplementary Information SI4; Supplementary Figure S2). Genetic variance is a prerequisite for selection (Barrett and Schluter, 2008).
Fitness of evolved strains was assessed by competing evolved stJS03 against stJS02. stJS02 is identical to stJS03, except that it is lacking $\mathrm{P}_{s S P E-2 \mathrm{G}^{-}}$ gfp and its associated chloramphenicol resistance gene. stJS03 can therefore be distinguished from stJS02 by spore-located GFP and chloramphenicol resistance. The fitness of stJS02 is comparable to that of stJS03 as determined beforehand by competition (Table 1).

Upon establishing increased fitness, single colonies of evolved stJS03 of each sampling time point were randomly picked from the appropriate plates. Evolved strains were labeled EvoR1, 2 or 3 when isolated from the regularly fluctuating environment after 24, 48 and $144 \mathrm{~h}$ (64 h after the final glucose addition), respectively, or EvoI1, 2 or 3 when obtained from the irregularly fluctuating environment (Supplementary Table S3).

\section{Whole-genome sequencing}

Evolved strains as well as ancestral strain stJS03 were sequenced. Single nucleotide polymorphisms (SNPs) and insertions/deletions (indel) of evolved siblings were identified by comparison with the parent strain.

\section{Constructing a tagE deletion strain}

Deletion of $\operatorname{tagE}$ was established by double homology crossover after transformation with a spectinomycin resistance cassette fused to the flanking regions of tagE. This cassette was obtained by PCR on genomic DNA (Supplementary Table S1) of a previously constructed tagE::spec strain (Allison et al., 2011) (gift of professor Eric Brown).

Swimming-motility assay

Swimming motility was tested by spotting $2.5 \mu \mathrm{l}$ of a $B$. subtilis-overnight-LB culture in the center of an LB plate, solidified with $0.3 \%$ Bacto agar. Pictures and colony sizes were recorded after overnight incubation at $30^{\circ} \mathrm{C}$ and after $48 \mathrm{~h}$ of additional incubation at room temperature.

\section{Results}

Determining appropriate glucose-feeding intervals Glucose consumption by B. subtilis 168 in CDSM shake-flask cultivations was determined (Supplementary Information SI5). Twenty millimolar of glucose is completely consumed after $\sim 17 \mathrm{~h}$ when starting with a low inoculum $(0.1 \% \mathrm{v} / \mathrm{v}$ overnight culture)(Supplementary Figure S3). Between glucose depletion and emergence of phase-bright spores, the number of live cells declines with $6.65 \%$ per hour (Supplementary Figure S4). In this shake-flask cultivation, $2 \mathrm{~h}$ after glucose depletion, over $78 \%$ of the cells survived. 
Table 1 Results of the competition experiments performed

\begin{tabular}{|c|c|c|c|c|c|}
\hline \multicolumn{2}{|c|}{ Competitions } & $4 \mathrm{~h}$ Regular & Irregular & Conclusion & SI no. \\
\hline stJS01 & $\Delta \operatorname{sig} F$ & & & $\begin{array}{l}\Delta s i g F \text { does not sporulate and loses the competition, } \\
\text { therefore spores increase fitness/the ability to sporulate } \\
\text { enhances survival }\end{array}$ & 7 \\
\hline stJS01 & NCIB3610 & & n.d. & $\begin{array}{l}\text { stJS01 loses the competition, therefore it is not optimally } \\
\text { adapted; good chance of selecting fitter mutants }\end{array}$ & 8 \\
\hline stJS02 & $\begin{array}{l}\text { stJS03 } \\
\text { (parental strain) }\end{array}$ & & & $\begin{array}{l}\text { Comparable fitness; stJS02 can serve as a reference for } \\
\text { fitness in competitions with evolved siblings of stJS03 }\end{array}$ & 9 \\
\hline stjS02 & $\begin{array}{l}\text { stJS03 } \\
\text { tagE::spec }\end{array}$ & & 12.8 & Loss of tagE increases fitness of stJS03 & 12 \\
\hline $\begin{array}{l}\text { stJS02 } \\
\text { tagE::spec }\end{array}$ & $\begin{array}{l}\text { stJS03 } \\
\text { (Parental strain) }\end{array}$ & & n.d. & Loss of $\operatorname{tag} E$ increases fitness of stJS02 & 13 \\
\hline
\end{tabular}

Abbreviation: n.d., not determined.

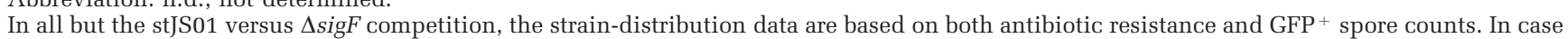
of stJS01 versus $\Delta s i g F$, antibiotic resistance was the selection criterion because $\Delta$ sig $F$ does not sporulate. The corresponding SI file number of the Excel files containing the raw data is given in the last column. Gene/locus names in the table are italicized.

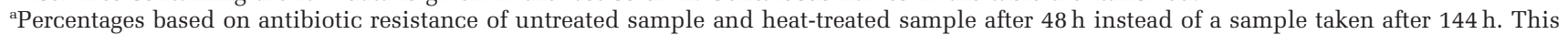
distribution was already reached after $24 \mathrm{~h}$.

${ }^{\mathrm{b}}$ Owing to low colony count in this antibiotic-resistance screen, these numbers are not significant. The percentage of GFP ${ }^{+}$spores (stJS03) was $46 \%$. For more solid evidence on the fitness advantage of tagE loss, the tagE::spec deletion was made in both backgrounds and competed with the tagE-positive versions of stJS02 and stJS03.

Phase-bright spores were observed from $\sim 19 \mathrm{~h}$ onwards, roughly $2 \mathrm{~h}$ after glucose depletion. Initiation of sporulation to spore maturation lasts $\sim 7 \mathrm{~h}$ (Hilbert and Piggot, 2004); thus, sporulation is initiated in part of the population before glucose is depleted. From these data we assume that $1 \mathrm{~mm}$ of glucose is consumed within $\sim 1 \mathrm{~h}$ (albeit dependent on biomass and portion of metabolically active cells) and that sporulation is by then induced in part of the population (Dixon and Spiegelman, 2002). A time frame of $4 \mathrm{~h}$ between glucose additions was chosen, so that sporulation would be initiated, while part of the vegetative cells should be able to survive this starvation episode in order to achieve selection pressure on the 'decision' to sporulate.

During batch fermentations, to which $1 \mathrm{~mm}$ glucose is sequentially applied, $\mathrm{pH}$ and $\mathrm{pO}_{2}$ both drop upon glucose influx (Figures 2a and b, top panel; Figure 2c), indicating that the culture starts respiring and becomes metabolically active. The recovery of the $\mathrm{pO}_{2}$ level (Figure 2c) shows that glucose was depleted before the next addition of glucose. Samples taken after $24 \mathrm{~h}$ of incubation from these batch fermentations, inoculated with vegetative cells, contained phase-bright spores (Figures 2a and $\mathrm{b}$, bottom panel), indicating that sporulation is initiated early in the culturing procedure.

The processes of sporulation initiation, spore maturation and germination take a minimum of $\sim 9 \mathrm{~h}: 7 \mathrm{~h}$ to form a spore (Hilbert and Piggot, 2004) and $1.5-2 \mathrm{~h}$ for $>90 \%$ of the spores to germinate under the conditions used in this study (Supplementary Information SI6; Supplementary Figures S5 and S6). As the full-length experiment lasts for $20 \mathrm{~h}$, such cycles can occur, causing the mixing of generations. Therefore, we do not present generation numbers.

Determining mid-experiment strain distributions proved difficult, as the time of sampling in relation to glucose influx influences the state of the cells. Therefore, we chose to present endpoint measurements and only use unambiguous mid-experiment data.

Competition experiment: the fluctuating environments select for sporulating strains

To test whether sporulation would be a fitnessincreasing strategy under the imposed selection 
a



b



$\mathrm{OD}_{600}$, Irregular

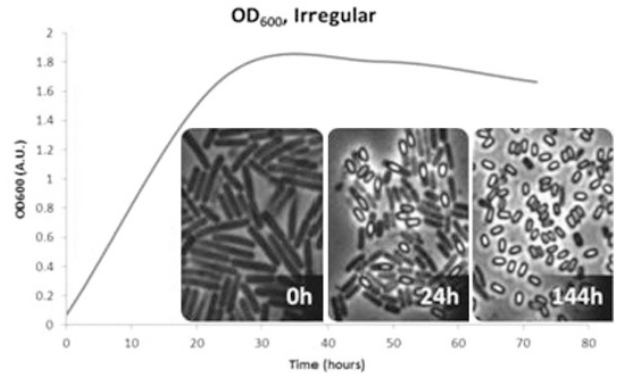

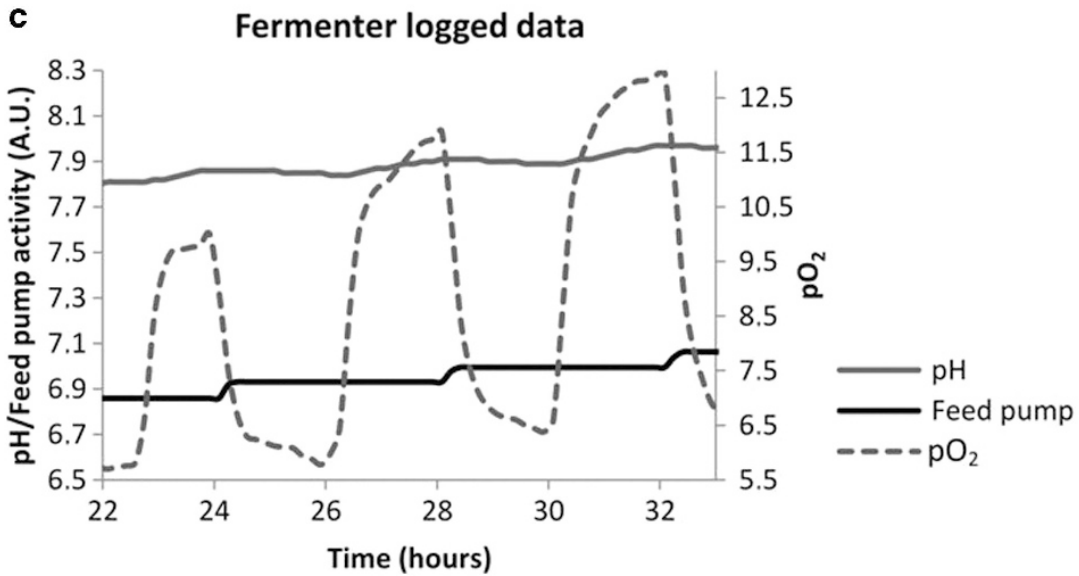

Figure 2 Typical graphs recorded during batch fermentations, in which glucose was added every $4 \mathrm{~h}$ (a) and at irregular intervals (b). Below the respective fluctuation regimes, the $\mathrm{OD}_{600}$ graphs are displayed including phase-contrast microscopy pictures of samples. (c) A detail plot of the same curve as shown in (a), now with $\mathrm{pO}_{2}$ values included. Every vertical jump of the feed pump line indicates addition of glucose, and at every glucose influx $\mathrm{pH}$ and $\mathrm{pO}_{2}$ drop as a result of metabolic activity of the culture. $\mathrm{pO} 2$ and $\mathrm{pH}$ recover before the next influx of glucose, illustrating that glucose is fully consumed between feeds.

pressure, a competition experiment between sporulating B. subtilis strain stJS01 and a non-sporulating $\Delta$ sigF strain was conducted (Supplementary Table S3). Sporulation causes loss of progenyproducing capacity and therefore lowers 'colonization potential' (Veening et al. 2008). $\sigma^{\mathrm{F}}$ is the earliest-acting regulatory protein in the forespore (Wang et al., 2006). sigF deletion results in arrested endospore formation after two asymmetrical septa are formed. The two forespore compartments can return to a viable vegetative form upon influx of nutrients, somewhat resembling binary fission (Dworkin and Losick, 2005). These characteristics make the $\Delta$ sigF strain an ideal competitor for testing whether sporulation is beneficial in comparison to remaining vegetative. This approach is preferred over tempering with the activity of master regulator for sporulation, Spo0A, of which the activity is intertwined with other regulatory processes and causes pleiotropic effects (Zuber and Losick, 1987; Chai et al., 2011). The logarithmic growth rates of both strains do not differ significantly in CDSM (Supplementary Figure S8).

The bioreactors were inoculated with exponentially growing cultures of strains stJS01 and $\Delta \operatorname{sigF}$ and subjected to the sequential glucose-feeding programs. Owing to the low selection pressure for sporulation it was feasible that $\Delta \operatorname{sig} F$ would outcompete the sporulating stJS01. Indeed, in the first half of these competitions $\Delta$ sigF proved fitter, but towards the end of the experiment stJS01 would outcompete $\Delta$ sigF (Table 1; Supplementary Information SI7). After 20 glucose additions with 4-h intervals, the proportion of the sporulating stJS01 strain increased to $>79 \%$, demonstrating that sporulation increases fitness over the course of 
20 glucose additions in CDSM with 4-h intervals. This trend was also observed in cultivations with irregular feeding intervals as well as in a pilot experiment with shorter, 3-h intervals (Table 1; Supplementary Information SI7).

Competition experiment: B. subtilis 168 is not optimally adapted for the designed environment; evolution of fitter mutants can be expected

In order for evolution to occur, the starting material should not be optimally adapted for the selection environment. To test this, a competition between $B$. subtilis stJS01 and wild isolate $B$. subtilis NCIB3610 (Kearns and Losick, 2003; Srivatsan et al., 2008) was performed. The natural environment of NCIB3610 is fundamentally less stable than laboratory conditions, and NCIB3610 should therefore perform better in the fluctuating selection environments. Indeed, after $48 \mathrm{~h},<6.5 \%$ of the population consisted of stJS01 (Table 1; Supplementary Information SI8). In CDSM the logarithmic growth rates were not significantly different (Supplementary Figure S9). Therefore, stJS01 is not optimally adapted for the imposed environmental fluctuations and evolutionary adaptation of a laboratory strain can be expected.

\section{Competition experiment: calibrating fitness of} ancestral strain stJSO3

Evolved stJS03 cannot be distinguished from ancestral stJS03 because of similar antibiotic resistance and fluorescence properties. Therefore, a replacement competitor with comparable fitness to stJS03 needs to be used. In contrast to stJS03, stJS02 does not contain $\mathrm{P}_{s s p E-2 \mathrm{G}}-g f p$. Therefore, stJS02 can be distinguished from stJS03 both visually and by antibiotic-resistance profiles. Fitness of stJS02 and stJS03 were compared in competition experiments. Sixty-four hours after the final glucose addition, the spore crops from the regularly and irregularly fluctuating environments contained $\sim 44$ and $\sim 46 \% \mathrm{GFP}^{+}$spores (stJS03), respectively (Table 1; Supplementary Information SI9; Supplementary Figure S10). This demonstrates that fitness of stJS02 and stJS03 are comparable in these environmentsin fact, stJS02 seems slightly fitter, probably because of lower maintenance for having one less constitutively expressed resistance marker-and thus stJS02 qualifies as a reference for fitness comparison with evolved strains.

Selection, identification and isolation of mutants with increased fitness

Spores of randomly mutagenized stJS03 were inoculated in the fluctuating environments for selection. This selection procedure was performed for two consecutive rounds (Figure 1, stage 3) without the presence of competitors. Spore crop, harvested after two selection cycles, was used in competition experiments with stJS02.
Spores of enriched selected UV-mutagenized stJS03 were mixed 1:1 (CFU) with spores of stJS02 and applied to the selection environment (Figure 1, stage 4). Samples taken during the competition contained an excess of green-fluorescent spores (Supplementary Figure S11) and the resulting spore crops contained over $72 \%$ of stJS03 spores $\left(\mathrm{Cm}^{\mathrm{r}}\right)$, demonstrating that the mixture of mutants contains genotypes with increased fitness (Supplementary Information SI10) Table 1.

\section{Whole-genome sequencing}

Observation of increased fitness of evolved stJS03 merited whole-genome sequencing of evolved siblings. Of each sample taken from the competition between stJS02 and evolved stJS03 (after 24, 48 and $144 \mathrm{~h}$ ), a single colony was isolated for wholegenome sequencing. These isolates are called EvoR1-3 (regularly fluctuating environment) and EvoI1-3 (irregularly fluctuating environment) (Supplementary Table S3). Comparison of the sequencing data of the selected mutant isolates and parental strain revealed EvoR1 and 3 to be isogenic and that all isolates, regardless the feeding regime, contained mutations in the tagE gene (Table 2). TagE is an UDP-glucose:polyglycerol phosphate glucosyltransferase that glycosylates glycerol phosphate subunits of major WTA using UDP-glucose. Evolved isolates EvoR2 and EvoI2 also carry mutations outside tagE (Table 2).

Non-tagE mutations in EvoR2. EvoR2 contains six SNPs and four deletions/insertions. No less than three mutations are found in tagE (Table 2). Three other mutations were found inside genes of unknown function (yesF, ycnC and $y w c I$ ) and one in an intergenic region. The remaining mutated genes are bmrU, yhaU and $\operatorname{sig} V$. bmrU is part of an operon-encoding multidrug transporter $\mathrm{Bmr}$ and regulator BmrR and is expressed, for example, under glucose starvation (Petersohn et al., 1999; Petersohn et al., 2001), yhaU encodes a $\mathrm{K}^{+} / \mathrm{H}^{+}$antiporter for $\mathrm{K}^{+}$efflux (Fujisawa et al., 2007) and $\operatorname{sig} V$ encodes one of seven extracytoplasmic function (ECF) sigma factors and is related to cell envelope homeostasis and antibiotic resistance (Mascher et al., 2007; Hashimoto et al., 2009, 2013; Matsuoka et al., 2011).

Non-tagE mutations in EvoI2. Evol2 contains, apart from an SNP in tagE, SNPs in ykuV and yusQ. The function of yus $Q$ is unknown, but ykuVencodes a hypothetical thiol:disulfide oxidoreductase, involved in protection of proteins against oxidative damage (Zhang et al., 2006).

Deletion of tagE is advantageous in an environment with fluctuating glucose availability

Four of the six evolved mutants contained only mutations in tagE, whereas all other mutations 
Table 2 SNPs and indels identified in evolved isolates compared to parent strain stJS03

\begin{tabular}{|c|c|c|c|c|c|}
\hline Feeding regime & Isolate & Mutation type & Mutated locus & Gene product function & AA changes \\
\hline & EvoR1 & SNP & $\operatorname{tag} E$ & $\begin{array}{l}\text { UDP-glucose:polyglycerol } \\
\text { phosphate glucosyltransferase }\end{array}$ & K360R \\
\hline & EvoR2 & SNP & $\operatorname{tag} E$ & & E471Q \\
\hline & & SNP & $\operatorname{tag} E$ & & V469L \\
\hline & & SNP & $\operatorname{sig} V$ & ECF sigma factor & A36T \\
\hline & & SNP & $y c n C$ & Unknown & G105V \\
\hline & & SNP & $y w c I$ & Unknown & A89P \\
\hline $4 \mathrm{~h}$ & & SNP & Intergenic & & \\
\hline \multirow[t]{6}{*}{ Regular intervals } & & Indel & $\operatorname{tag} E$ & & V469 frameshift \\
\hline & & Indel & $b m r U$ & Hypothetical multidrug-resistance protein & N18 frameshift \\
\hline & & Indel & yesF & unknown & M144 frameshift \\
\hline & & Indel & yhaU & $\mathrm{K}^{+} / \mathrm{H}^{+}$antiporter for $\mathrm{K}^{+}$efflux & G277 frameshift \\
\hline & EvoR3 & SNP & $\operatorname{tag} E$ & & K360R \\
\hline & EvoI1 & SNP & $\operatorname{tag} E$ & & Q297 stop codon \\
\hline \multirow[t]{4}{*}{ Irregular intervals } & Evol2 & SNP & $\operatorname{tag} E$ & & L436P \\
\hline & & SNP & ykuV & Thiol:disulfide oxidoreductase & D89Y \\
\hline & & SNP & yusQ & Unknown & E31K \\
\hline & Evol3 & Indel & $\operatorname{tag} E$ & & E161 frameshift \\
\hline
\end{tabular}

Abbreviation: SNP, Single nucleotide polymorphism.

Included are the specific amino-acid changes per mutated gene. Lists of all identified SNPs and indels can be found in Supplementary

Information SI11. Gene/locus names in the table are italicized. The commonly mutated tagE locus is highlighted in bold.

found were unique (Table 2). Three of the six mutated tagE genes contain either a frameshift or a novel stop codon, preventing the production of a functional TagE. This merits the assumption that loss of functional TagE is the major beneficial mutation under the selection pressures applied. To confirm that the loss of tagE is advantageous, $\operatorname{tag} E$ was replaced by a spectinomycin resistance cassette in stJS03. The resulting strain competed against stJS02 in both the regular and irregular glucose-fluctuating environments. As expected, the resulting spore crops contained 76-82\% GFP ${ }^{+}$spores (stJS03 tagE::spec) and resulted in $85 \%$ Spec $^{\mathrm{r}}$ colonies after heat treatment (Table 1).

The antibiotic-based fitness comparison of stJS02 versus stJS03 in the 4-h regular fluctuating environment had low statistical reliability as a result of low colony numbers (Supplementary Information SI9). As an additional control tagE was deleted from stJS02, the reference strain for fitness. This strain competed against ancestral strain stJS03 resulting in $\sim 70 \%$ stJS02 tagE::spec spores, corroborating the fitness advantage of the loss of tagE under these conditions.

As a negative control, both stJS02 and stJS03 tagE::spec were co-inoculated 1:1 $\left(\mathrm{OD}_{600}\right)$ in CDSM shake flasks initially containing $20 \mathrm{~mm}$ glucose. This condition mimics the batch-fermenter situation without the presence of starvation bottlenecks. These experiments resulted in spore crops containing 36\% stJS03 tagE::spec spores (Supplementary Figure S12). This demonstrates that tagE loss is beneficial only under repetitive glucose-limitation conditions (Supplementary Information SI14).
Deletion of tagE affects the timing of differentiation B. subtilis strain stJS03 tagE::spec was studied for altered differentiation properties. The growth curves of stJS03 and stJS03 tagE::spec were not significantly different, nor were significant differences detected in spore accumulation throughout a growth curve (data not shown). Spore accumulation was studied by microscopically investigating shake-flask samples and by means of time-lapse microscopy (de Jong et al., 2011a). Germination of spores of either strain was studied spectrophotometrically and using time-lapse microscopy, but was also not aberrant.

Swimming motility. Only when the three master regulators that control differentiation-DegU, ComA and Spo0A-are inactive, B. subtilis can differentiate into motile cells (Lopez and Kolter, 2010). A clear reduction in swimming-based migration is observed for stJS03 tagE::spec after overnight cultivation at $37^{\circ} \mathrm{C}$ on $\mathrm{LB}$ supplemented with $0.3 \%$ agar (Figure 3a), which indicates modified master regulator responses. Additionally, after 48 more hours at room temperature, at the edge of the stJS03 tagE::spec colony granule-like structures formed (Figures 3a and b). These granules are brightly green fluorescent relative to the rest of the colony, indicating the presence of spores (Figure 3c). Indeed, when such a granule is extracted from the plate using a micropipette tip and applied onto a microscope slide, spores can be found (Figure 3d). This phenotype was also observed for all evolved mutants (Supplementary Information SI15; Supplementary Figures S13 and S14) and could be complemented using an integrative vector containing tagE downstream of a $\mathrm{P}_{\text {spank }}$ promoter 

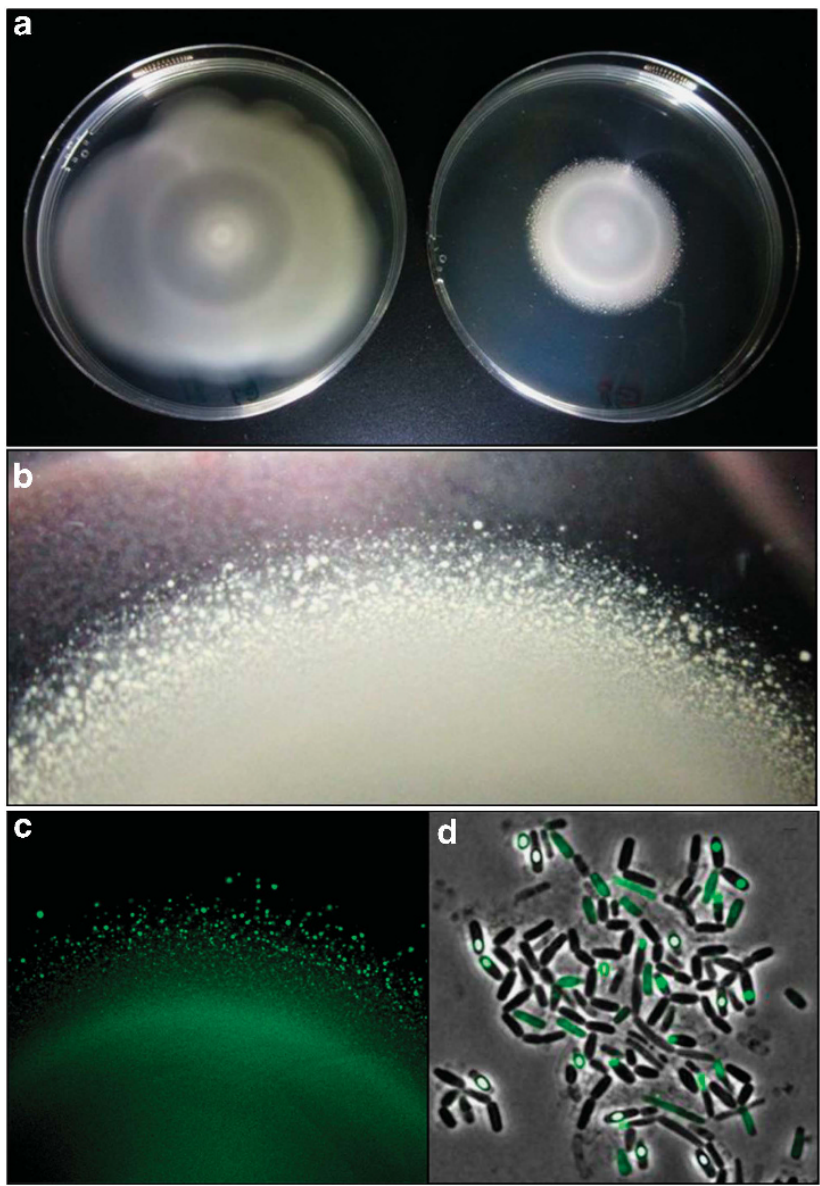

Figure 3 Swimming motility was compared using LB plates solidified with $0.3 \%$ agar. (a) The petridish on the left was inoculated with stJS03, the right one with stJS03 tagE::spec. (b) Detail of the edge of the stJS03 tagE::spec colony. (c) A microscope image of the green fluorescence of a stJS03 tagE::spec colony grown on $0.3 \%$ LB agar plate displaying high fluorescence for the extra-colonial granules. (d) A phase-contrast microscopy image of cells extracted from an extra-colonial granule, with the GFP signal overlayed and false colored green.

(Supplementary Information SI16; Supplementary Figure S15).

\section{Discussion}

We studied evolutionary adaptation of $B$. subtilis in response to repetitive starvation episodes. One millimolar glucose was fed with intervals of $4 \mathrm{~h}$, which was long enough for the offered glucose to be fully consumed and starvation to occur (Figure 2). In a second evolution line, feeding intervals were randomized, but the total length of the experiment remained $80 \mathrm{~h}$.

Although the famine was not severe (over $75 \%$ of vegetative cells are assumed to survive; Supplementary Information SI5), sporulation was still advantageous as demonstrated by the victory of sporulating $B$. subtilis strain stJS01 over a

non-sporulating $\triangle \operatorname{sig} F$ strain in a competition experiment (Table 1; Supplementary Information SI7). Initially $\Delta s i g F$ seemed to outcompete stJS01, but towards the end stJS01 would prevail. Sporulation involves an fitness cost, but we hypothesize that spores increase colonization potential upon re-entering the reproductive cycle.

Competition experiments between $B$. subtilis laboratory strain stJS01 and wild isolate NCIB3610 showed the superior fitness of the NCIB3610 in our set-up (Supplementary Information SI8). This was expected because of the fundamentally more variable environment to which NCIB3610 adapted in contrast to laboratory conditions that induce phenotypical deterioration (Maughan et al., 2006). The ancestral laboratory strain is thus not optimally adapted to this environment, therefore mutants with increased fitness could be expected. That fitter mutants indeed emerged became evident when evolved siblings of laboratory strain stJS03 outcompeted 'reference for fitness' strain stJS02: a surplus of green-fluorescent stJS03 spores was found already $24 \mathrm{~h}$ after inoculation (Supplementary Figure S11). From these competitions, evolved mutants were isolated and their genomes sequenced.

One question we wanted to address was whether unpredictability of the environmental fluctuations - a situation closer resembling natural environments-would result in different selection pressures and thus different mutations. In unpredictably fluctuating environments, fitness can be increased by spreading risks using strategies such as bet-hedging. Bet-hedging can be achieved by, for example, stochastic-phenotype switching and contrasts with deterministic responses predicted to be more favorable for predictable environmental fluctuations (Simons, 2009, 2011; de Jong et al., 2011b). When comparing mutations between the isolates from either the regularly or irregularly fluctuating environment, a clear distinction could not be made (Table 2). We hypothesize that the fluctuation frequency was too high for differentiation of selection pressures between both feeding regimes. Strikingly, regardless the feeding regime, all isolates contain a mutated tagE gene that encodes a major WTA-modifying enzyme. This shows the robust fitness advantage of mutations in tagE under the imposed selection conditions.

Evolved strains EvoR2 and EvoI2 (isolated after $48 \mathrm{~h}$ from the regularly- and irregularly fluctuating environments, respectively) were the only isolates that contain unique, non-tagE mutations (Table 2), of which bmrU, yhaU, and $\operatorname{sig} V$ (EvoR2) and ykuV (EvoI2) encode for a protein with a known or hypothetical function.

In EvoR2, bmrU and yhaU contain indels, therefore the YhaU/YhaT antiporter function, as well as potential multidrug resistance exerted by Bmr, is lost (Fujisawa et al., 2007). Hypothetical protein BmrU, however, may not be essential for this 
multidrug-resistance system (Petersohn et al., 1999; Petersohn et al., 2001), therefore it may be a phenotypically silent mutation. yku (encoding a hypothetical thiol:disulfide oxidoreductase) and $\operatorname{sig} V$ contain SNPs, therefore it is uncertain how the functions of these proteins are affected. Nevertheless, the $\operatorname{sig} V$ mutation is interesting because $\operatorname{sig} V$ is expressed upon cell wall stress (Hashimoto et al., 2009; Hashimoto et al., 2013), perhaps induced by malfunctioning TagE. Although it has been suggested that ECF sigma factor $\sigma^{\mathrm{V}}$ is functionally redundant because of regulon overlap (Mascher et al., 2007), mutations tuning $\sigma^{\mathrm{V}}$ affinity may still be beneficial. Without detailed expression data, however, discussing fitness advantage of these non$\operatorname{tag} E$ mutations is merely speculation; the genes may be silent under the imposed culturing conditions.

Because endpoint mutants were sequenced, information on the emergence of mutations in time is lacking. Therefore, we cannot exclude genetic hitchhiking nor a scenario where mutations emerged spontaneously as a result of mutated tagE, or vice versa. These mutations may or may not be fixed in the population, but randomly picking three strains from a mixture of fitter mutants holds no mutation frequency information. However, EvoR1 and EvoR3 are isogenic and for them to be randomly picked from two different samples, extracted at two distinct time points hints towards this being a dominant genotype in the regular glucose-feeding line. It would be highly interesting to sequence many more strains from the fitter mutant batch and continue selection in either environment using the current batch of evolved strains as starting material-or the tagE deletion strain-to address these topics.

In four of the six isolates, tagE was the only mutated gene and three of the six mutated tagE genes contain either a frameshift or a novel stop codon (Table 2). This suggests that the major beneficial effect of the mutations is loss of functional TagE. Indeed, in competitions, tagE deletion stJS03 tagE::spec proved more competitive than stJS03 (Table 2; Supplementary Information SI9, SI12), confirming our hypothesis. Moreover, stJS03 tagE::spec displayed the same altered phenotype as the evolved mutants (Figure 3; Supplementary Information SI15).

tagE is a non-essential gene encoding a protein that glycosylates glycerol phosphate subunits of WTA, using UDP-glucose as a substrate. The physiological function of this modification is yet unknown (Allison et al., 2011). Together with lipoteichoic acids, WTA accounts for over $60 \%$ of the mass of the Gram-positive cell wall. Of the poly(glycerol phosphate) subunits of WTA, 25\% are glycosylated (Archibald et al., 1969; D’Elia et al., 2006; Silhavy et al., 2010). A logical explanation for increased fitness by loss of tagE would be economical; it would save a considerable amount of glucose from being incorporated in the cell wall (Dixon and
Spiegelman, 2002). However, isolates of evolved stJS03 and stJS03 tagE::spec did not display significantly different logarithmic growth rates, although different growth dynamics may apply under the fluctuating culturing conditions. These strains do travel considerably less far in swimmingmotility assays and develop granule-like structures at the colony edge earlier than the ancestral strain stJS03 (Figure 3). This suggests altered responses of the master regulators that govern cell differentiation in relation to the environmental nutrient state (Lopez and Kolter, 2010).

Only the swimming-motility assays proved sensitive enough for significant and reproducible detection of the minute changes in differentiation timing caused by TagE loss. Assays for studying growth rate, sporulation initiation, spore accumulation and spore germination revealed no significant differences between stJS03 and stJS03 tagE::spec. Therefore, we can only conclude that initiation of differentiation is altered, without hypothesizing on altered timing of specific differentiation programs such as sporulation.

All evolved stJS03 isolates display the same phenotype as stJS03 tagE::spec (Supplementary Figure S13). This implies that the amino-acid substitutions in TagE of EvoR1, EvoR3 and EvoI2 are detrimental for its function. This could be expected from the L436P substitution in EvoI2, where the proline may act as a secondary-structure breaker (Li et al., 1996). Apparently, a K360R aminoacid substitution is also detrimental, and therefore this amino acid may be crucial for TagE activity (for example, active site located).

Reduced swimming motility and altered differentiation timing in relation to environmental nutrient state is counterintuitive with the hypothesis that tagE-negative strains utilize glucose more efficiently and consequently starve later. It is also unlikely that this phenotype can be solely accounted for by altered modification of cell wall components. Recently, it has been shown that UDP-glucose, the substrate of TagE, serves as an intracellular proxy for nutrient availability involved in coordinating growth rate and cell division (Weart et al., 2007; Chien et al., 2012). Loss of TagE causes altered UDP-glucose levels and consequential disproportionate nutrient availability read-outs. It is fair to assume that this system also affects the development of phenotypes that have evolved for the purpose of surviving adverse times. So far, it is not fully understood how B. subtilis coordinates sporulation by nutrient-limitation sensing (Dixon and Spiegelman, 2002; Maughan and Nicholson, 2004; Fujita and Losick, 2005; Eswaramoorthy et al., 2011) and we propose a role for UDP-glucose in this process. Interestingly, $\operatorname{sig} V$, a mutated gene in EvoR2, is expressed upon deletion of ugtP (Matsuoka et al., 2011), an enzyme that exerts its function upon binding UDP-glucose. Perhaps internal UDP-glucose levels influence sig $V$ expression, 
which may reduce fitness in our selection procedure, making mutations in $\operatorname{sig} V$ beneficial.

It is interesting to find mutations in genes of which the importance is (still) under debate in the evolved strains. Sporulation is a high cost, irreversible pathway, with an undeniable burden on propagation potential, therefore waiting for environmental cues with the chance of running out of energy before spore finalization becomes too risky. Initiation of sporulation before glucose has been depleted (Supplementary Information SI5) shows the occurrence of stochastic onset of sporulation, a beneficial strategy to cope with uncertainty of the future environment and probably caused by intracellular noise. Intracellular noise has been predicted to be insensitive to selection pressure, and previously experimental evolution with strong selection for sporulation in $B$. subtilis did not result in evolved strains with enhanced sporulation efficiency (Maughan and Nicholson, 2004). This suggested that intracellular noise is a key element embedded in the regulatory network for sporulation. One way to then 'trick' the regulatory network to respond differently is to modify its input and affect environmental nutrient state read-outs, for example, by altering intracellular UDP-glucose levels.

The study presented here shows the powerful possibilities of experimental evolution and competition-based fitness determination. It is highly interesting that mutations in a single, seemingly redundant, gene can generate a profound fitness advantage. Additionally, the specific selection against tagE and/or the function of its product may help to elucidate the physiological function of this enzyme.

\section{Conflict of Interest}

The authors declare no conflict of interest.

\section{Acknowledgements}

We thank Wout Overkamp for providing B. subtilis $\Delta$ sigF and for valuable help with the bioreactors. Martijn Herber is thanked for helping programming the bioreactor software. Professor Eric Brown is thanked for providing B. subtilis $\triangle$ tagE and valuable discussion and Mirjam Boonstra for the kind gift of plasmid pMB002. Pieter van der Vlies (UMCG) is much appreciated for his help with the analysis of the genome-sequencing data. Dr Dirk-Jan Scheffers, Dr Sander Hille and Dr Michael Emmerich are thanked for their valuable discussions. Dr Jan-Willem Veening is thanked for valuable discussions and critically reading this manuscript. Anja Ridder and Dr Morten Kjos are thanked for critically reading the manuscript. Work in the laboratory of OPK is supported by the research program of the Kluyver Centre for Genomics of Industrial Fermentation, which is part of the Netherlands Genomics Initiative/Netherlands Organization for Scientific Research. JS and FDV were funded by the NWO Computational Life Sciences Program, projectnr. 600.635.100.08N26.

\section{References}

Allison SE, D’Elia MA, Arar S, Monteiro MA, Brown ED. (2011). Studies of the genetics, function, and kinetic mechanism of TagE, the wall teichoic acid glycosyltransferase in Bacillus subtilis 168. J Biol Chem 286: 23708-23716.

Archibald AR, Baddiley J, Heptinstall S. (1969). The distribution of the glucosyl substituents along the chain of the teichoic acid in walls of Lactobacillus buchneri N.C.I.B. 8007. Biochem J 111: $245-246$.

Barrett RD, Schluter D. (2008). Adaptation from standing genetic variation. Trends Ecol Evol 23: 38-44.

Barrick JE, Yu DS, Yoon SH, Jeong H, Oh TK, Schneider D et al. (2009). Genome evolution and adaptation in a long-term experiment with Escherichia coli. Nature 461: 1243-1247.

Beaumont HJ, Gallie J, Kost C, Ferguson GC, Rainey PB. (2009). Experimental evolution of bet hedging. Nature 462: 90-93.

Blount ZD, Barrick JE, Davidson CJ, Lenski RE. (2012). Genomic analysis of a key innovation in an experimental Escherichia coli population. Nature 489: 513-518.

Chai Y, Norman T, Kolter R, Losick R. (2011). Evidence that metabolism and chromosome copy number control mutually exclusive cell fates in Bacillus subtilis. EMBO J 30: 1402-1413.

Chien AC, Hill NS, Levin PA. (2012). Cell size control in bacteria. Curr Biol 22: R340-R349.

D’Elia MA, Millar KE, Beveridge TJ, Brown ED. (2006). Wall teichoic acid polymers are dispensable for cell viability in Bacillus subtilis. J Bacteriol 188: 8313-8316.

de Jong IG, Beilharz K, Kuipers OP, Veening JW. (2011a). Live cell imaging of Bacillus subtilis and Streptococcus pneumoniae using automated time-lapse microscopy. J Vis Exp 53: pii 3145.

de Jong IG, Haccou P, Kuipers OP. (2011b). Bet hedging or not? A guide to proper classification of microbial survival strategies. Bioessays 33: 215-223.

Dixon LG, Spiegelman GB. (2002). Glucose-resistant sporulation in Bacillus subtilis crsA47 mutants does not depend on promoter switching at the spooA gene. J Bacteriol 184: 1458-1461.

Dworkin J, Losick R. (2005). Developmental commitment in a bacterium. Cell 121: 401-409.

Errington J. (1993). Bacillus subtilis sporulation: regulation of gene expression and control of morphogenesis. Microbiol Rev 57: 1-33.

Eswaramoorthy P, Dravis A, Devi SN, Vishnoi M, Dao HA, Fujita M. (2011). Expression level of a chimeric kinase governs entry into sporulation in Bacillus subtilis. J Bacteriol 193: 6113-6122.

Fujisawa M, Ito M, Krulwich TA. (2007). Three twocomponent transporters with channel-like properties have monovalent cation/proton antiport activity. Proc Natl Acad Sci USA 104: 13289-13294.

Fujita M, Gonzalez-Pastor JE, Losick R. (2005). High- and low-threshold genes in the Spo0A regulon of Bacillus subtilis. J Bacteriol 187: 1357-1368.

Fujita M, Losick R. (2005). Evidence that entry into sporulation in Bacillus subtilis is governed by a gradual increase in the level and activity of the master regulator Spo0A. Genes Dev 19: 2236-2244. 
Gordo I, Perfeito L, Sousa A. (2011). Fitness effects of mutations in bacteria. J Mol Microbiol Biotechnol 21: 20-35.

Hageman JH, Shankweiler GW, Wall PR, Franich K, McCowan GW, Cauble SM et al. (1984). Single, chemically defined sporulation medium for Bacillus subtilis: growth, sporulation, and extracellular protease production. J Bacteriol 160: 438-441.

Hashimoto M, Takahashi H, Hara Y, Hara H, Asai K, Sadaie Y et al. (2009). Induction of extracytoplasmic function sigma factors in Bacillus subtilis cells with membranes of reduced phosphatidylglycerol content. Genes Genet Syst 84: 191-198.

Hashimoto M, Seki T, Matsuoka S, Hara H, Asai K, Sadaie Y et al. (2013). Induction of extracytoplasmic function sigma factors in Bacillus subtilis cells with defects in lipoteichoic acid synthesis. Microbiology 159: $23-35$.

Hilbert DW, Piggot PJ. (2004). Compartmentalization of gene expression during Bacillus subtilis spore formation. Microbiol Mol Biol Rev 68: 234-262.

Hoch JA. (1993). Regulation of the phosphorelay and the initiation of sporulation in Bacillus subtilis. Annu Rev Microbiol 47: 441-465.

Kearns DB, Losick R. (2003). Swarming motility in undomesticated Bacillus subtilis. Mol Microbiol 49: $581-590$

Lemon KP, Kurtser I, Wu J, Grossman AD. (2000). Control of initiation of sporulation by replication initiation genes in Bacillus subtilis. J Bacteriol 182 2989-2991.

Li SC, Goto NK, Williams KA, Deber CM. (1996). Alphahelical, but not beta-sheet, propensity of proline is determined by peptide environment. Proc Natl Acad Sci USA 93: 6676-6681.

Lopez D, Kolter R. (2010). Extracellular signals that define distinct and coexisting cell fates in Bacillus subtilis. FEMS Microbiol Rev 34: 134-149.

Mascher T, Hachmann AB, Helmann JD. (2007). Regulatory overlap and functional redundancy among Bacillus subtilis extracytoplasmic function sigma factors. J Bacteriol 189: 6919-6927.

Matsuoka S, Chiba M, Tanimura Y, Hashimoto M, Hara H, Matsumoto K. (2011). Abnormal morphology of Bacillus subtilis ugtP mutant cells lacking glucolipids. Genes Genet Syst 86: 295-304.

Maughan H, Nicholson WL. (2004). Stochastic processes influence stationary-phase decisions in Bacillus subtilis. J Bacteriol 186: 2212-2214.

Maughan H, Callicotte V, Hancock A, Birky CW Jr., Nicholson WL, Masel J. (2006). The population genetics of phenotypic deterioration in experimental populations of Bacillus subtilis. Evolution 60: 686-695.

Petersohn A, Antelmann H, Gerth U, Hecker M. (1999). Identification and transcriptional analysis of new members of the sigmaB regulon in Bacillus subtilis. Microbiology 145(Pt 4): 869-880.

Petersohn A, Brigulla M, Haas S, Hoheisel JD, Volker U, Hecker M. (2001). Global analysis of the general stress response of Bacillus subtilis. J Bacteriol 183: 5617-5631.

Rainey PB, Beaumont HJ, Ferguson GC, Gallie J, Kost C, Libby E et al. (2011). The evolutionary emergence of stochastic phenotype switching in bacteria. Microb Cell Fact 10(Suppl 1): S14.

Silhavy TJ, Kahne D, Walker S. (2010). The bacterial cell envelope. Cold Spring Harb Perspect Biol 2: a000414.

Simons AM. (2009). Fluctuating natural selection accounts for the evolution of diversification bet hedging. Proc Biol Sci 276: 1987-1992.

Simons AM. (2011). Modes of response to environmental change and the elusive empirical evidence for bet hedging. Proc Biol Sci 278: 1601-1609.

Sonenshein AL. (2000). Control of sporulation initiation in Bacillus subtilis. Curr Opin Microbiol 3: 561-566.

Srivatsan A, Han Y, Peng J, Tehranchi AK, Gibbs R, Wang JD et al. (2008). High-precision, whole-genome sequencing of laboratory strains facilitates genetic studies. PLoS Genet 4: e1000139.

Veening JW, Hamoen LW, Kuipers OP. (2005). Phosphatases modulate the bistable sporulation gene expression pattern in Bacillus subtilis. Mol Microbiol 56: 1481-1494.

Veening JW, Smits WK, Hamoen LW, Kuipers OP. (2006). Single cell analysis of gene expression patterns of competence development and initiation of sporulation in Bacillus subtilis grown on chemically defined media. J Appl Microbiol 101: 531-541.

Veening JW, Stewart EJ, Berngruber TW, Taddei F, Kuipers OP, Hamoen LW. (2008). Bet-hedging and epigenetic inheritance in bacterial cell development. Proc Natl Acad Sci USA 105: 4393-4398.

Wang ST, Setlow B, Conlon EM, Lyon JL, Imamura D, Sato $\mathrm{T}$ et al. (2006). The forespore line of gene expression in Bacillus subtilis. J Mol Biol 358: 16-37.

Weart RB, Lee AH, Chien AC, Haeusser DP, Hill NS, Levin PA. (2007). A metabolic sensor governing cell size in bacteria. Cell 130: 335-347.

Webb CD, Decatur A, Teleman A, Losick R. (1995). Use of green fluorescent protein for visualization of cellspecific gene expression and subcellular protein localization during sporulation in Bacillus subtilis. J Bacteriol 177: 5906-5911.

Zhang X, Hu Y, Guo X, Lescop E, Li Y, Xia B et al. (2006). The Bacillus subtilis $\mathrm{YkuV}$ is a thiol:disulfide oxidoreductase revealed by its redox structures and activity. J Biol Chem 281: 8296-8304.

Zuber P, Losick R. (1987). Role of AbrB in Spo0A- and Spo0B-dependent utilization of a sporulation promoter in Bacillus subtilis. J Bacteriol 169: 2223-2230.

Supplementary Information accompanies this paper on The ISME Journal website (http://www.nature.com/ismej) 